\title{
46,XX Gonadal Dysgenesis
}

National Cancer Institute

\section{Source}

National Cancer Institute. 46,XX Gonadal Dysgenesis. NCI Thesaurus. Code C120197.

Gonadal dysgenesis in an individual with 46,XX karyotype and female phenotype. 